\title{
Role of Neural Network in Healthcare System
}

\author{
Kiran Bala Dubey \\ Assistant Professor Computer Science, \\ Govt. Nagarjuna P.G. College of Science, Raipur
}

\author{
Gyanesh Shrivastava \\ Professor \& Head, School of IT, \\ MATS University, Raipur
}

\begin{abstract}
Healthcare is the acquisition area in our today's necessity. Healthcare costs approximately the world is on the outgrowth, creating a strong necessity for new ways of supporting the need of the healthcare system. Neural networks have been evidently found in many promising applications of the healthcare domain. Neural Network is utilized as a knowledge discovery proficiency to identify the service quality factors that are important to patients.
\end{abstract}

Neural networks have parallel computing devices, which are fundamentally an activity to form a computer framework of the human brain. The primary objective of neural network is to create a system to do a variety of computational tasks quicker than the traditional systems. A neural network is utilized to learn pattern and relationship in data.

The purpose of this paper is to provide an exposure of the application of neural network systems in healthcare system. The Neural Network is a network of artificial neurons, same as found in human brains, for resolving artificial intelligence problems like pattern recognition, image identification, data compression, classification and optimization.

\section{Keywords}

Neural Network, knowledge discovery, artificial intelligence

\section{INTRODUCTION}

An Artificial Neuron Network (ANN), usually known as neural network is a computing system made-up of a number of simple, extremely interconnected processing elements, which process information through their impulsive state reply to outer inputs. Neural network is a computational structure dependent on the structure and utilization of natural neural networks. It correlates with an artificial human nervous system for getting, processing, and sending information in terms of computer system.

The idea of ANN depends on the possibility that workings of human brain by constituent the correct connection, can be reproduced using silicon and wires as living neurons and dendrites.

Neurons are 86 billion nerve cells that forms the human brain. They are linked to other millions of cells by Axons. Dendrites accept improvements from external environment or inputs obtained from sensory organs. These data sources make electric impulses which quickly move through the neural network.

A neuron can then pass the message to other neuron to manage the issue.

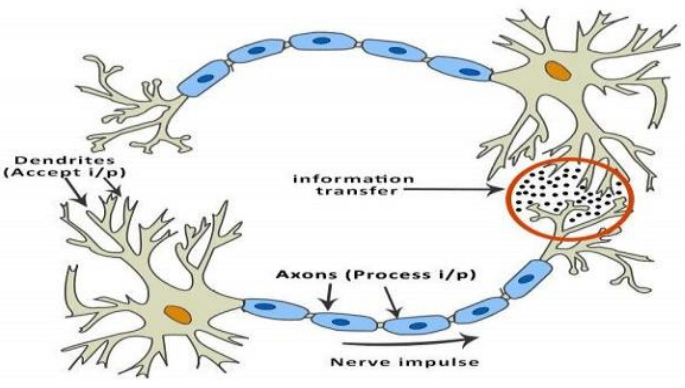

Fig. 1 Biological neural network

ANNs are comprised of aggregate nodes, which is like biological neurons of human brain. The neurons are associated by links and they are related with each other. Simple operations can be executed on the input data received from the input nodes then the outcome of these operations is transferred to different neurons. The end product of every node is called its node value or stimulation.

Every link is attached with weight. ANNs are capable of learning, which happens by variable weights. The following diagram demonstrates a simple ANN -

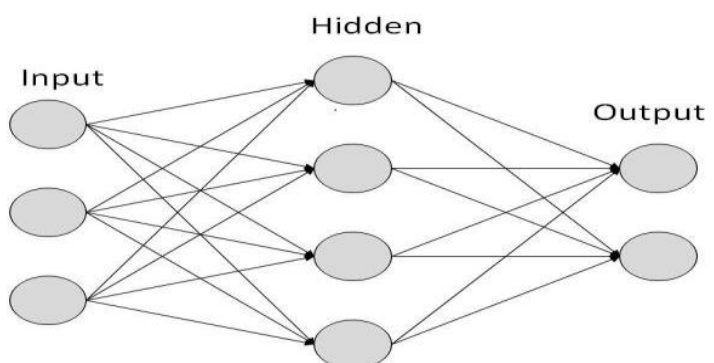

Fig. 2 Artificial neural network

In general, there are three kind of layers in an artificial neural network:-

1. Input Layer - All the inputs are get into the network by input layer. The Input layer communicates with the external atmosphere. Its responsibility is to manage all the inputs as it were. These inputs are then passed to the hidden layers. The input layer should design the condition for which we are setting up the neural network. Each input neuron should represent for some autonomous variable that has an effect over the output of the neural network.

2. Hidden Layers - The hidden layer is made up of set of neurons that has activation function. It is an intermediate layer found between the input layer and the output layer. Its capability is to manage the sources of input got from input layer. The number of hidden layer is depending on the degree of accuracy required.

3. Output Layer - After processing the information is open at the output layer. The output layer of an artificial neural 
network aggregate and give the information as a way it has been needed to give. The number of neurons in output layer should be authentically related with the capacity of the neural network.

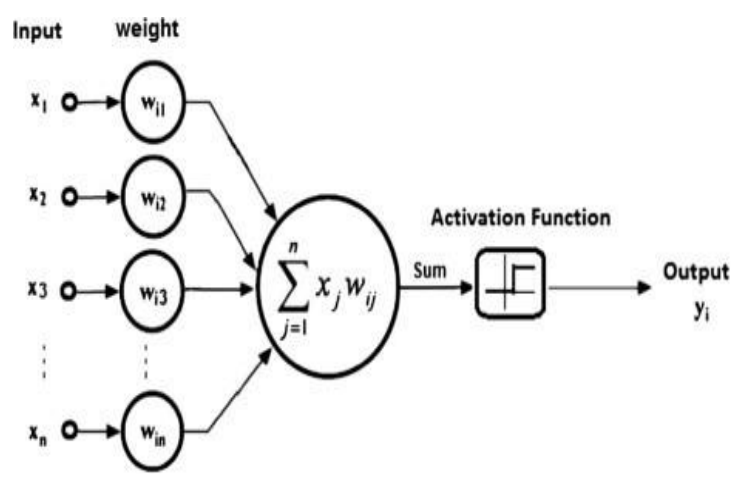

Fig.3 showing the Activation functions for ANN [2]

Summation function $=\mathrm{X}_{1} \mathrm{Wi}_{1}+\mathrm{X}_{2} \mathrm{Wi}_{2}+\ldots+\mathrm{X}_{\mathrm{n}} \mathrm{Wi}_{\mathrm{n}}$

The benefits of neural Networks:

ANNs have some important benefits that make it more suitable for assured problems and situations:

1. ANNs have the ability to process huge amount of data.

2. ANNs have power to learn and represent non-linear and complex associations between inputs and outputs.

3. ANNs can generalize means after learning from the initial inputs and their associations, it can conclude unseen associations on hidden data also, thus producing the model generalize and predict on hidden data.

4. ANN has ability to find out hidden associations within the Data with high unpredictability and non-constant variance without forcing any fixed associations within the data. This is often very useful in financial forecasting (e.g. stock prices) where data volatility is extremely high.

\section{A few roles:}

1. Image Processing, speech recognition and Character recognition: Speech recognition is a natural language processing system. ANNs are assuming a significant function in image and character recognition. Character recognition, for example, handwriting has many applications in fraud detection and even open security evaluate. Image recognition is an emerging field with wide applications from facial acknowledgment in social media, cancer detection in medicine, satellite imagery handling for agricultural and defence usage.

2. Forecasting: Forecasting is required broadly in routine business decisions to make economic and financial policy.

3. ANNs used as a powerful tool to help physicians to diagnose disease [7].

ANNs are powerful prototypes that have a broad range of roles. Above, we have listed a few important ones, but it has large roles on many different fields in security, medicine, banking, agriculture, finance and defence.

\section{BIOLOGICAL VS. ARTIFICIAL NEURAL NETWORK}

The main differences between biological and artificial neural network are -

\begin{tabular}{|c|c|c|}
\hline Criteria & $\begin{array}{l}\text { Biological Neural } \\
\text { Network }\end{array}$ & $\begin{array}{ll}\text { Artificial } & \text { Neural } \\
\text { Network } & \end{array}$ \\
\hline Processing & $\begin{array}{l}\text { Extremely } \\
\text { operations, slow } \\
\text { but superior than } \\
\text { Artificial Neural } \\
\text { Network. } \\
\end{array}$ & $\begin{array}{lr}\text { Extremely } & \text { parallel } \\
\text { operations, fast but } \\
\text { inferior r than } \\
\text { Biological } & \text { Neural } \\
\text { Network. } & \\
\end{array}$ \\
\hline $\begin{array}{l}\text { Size \& } \\
\text { complexity }\end{array}$ & $\begin{array}{l}\text { The number of } \\
\text { neurons in the brain } \\
\text { is computation to } \\
\text { about } 10^{11} \text { neurons } \\
\text { and } 10^{15} \text { link. The } \\
\text { size \& complexity of } \\
\text { link gives the brain } \\
\text { the power of } \\
\text { performing complex } \\
\text { pattern recognition } \\
\text { tasks, which cannot } \\
\text { be realized on a } \\
\text { computer. }\end{array}$ & $\begin{array}{l}10^{2} \text { to } \\
10^{4} \text { nodes mainly } \\
\text { depend on the type of } \\
\text { application and } \\
\text { network designer. }\end{array}$ \\
\hline $\begin{array}{l}\text { Storage } \\
\text { capacity }\end{array}$ & $\begin{array}{l}\text { Stores the } \\
\text { information in the } \\
\text { capability of the } \\
\text { interconnections }\end{array}$ & $\begin{array}{l}\text { Stores the information } \\
\text { in the memory } \\
\text { locations }\end{array}$ \\
\hline Speed & $\begin{array}{l}\text { Biological neurons } \\
\text { are slow in } \\
\text { processing } \\
\text { information. }\end{array}$ & $\begin{array}{l}\text { Neural networks are } \\
\text { quicker in processing } \\
\text { information, }\end{array}$ \\
\hline $\begin{array}{l}\text { Control } \\
\text { mechanism }\end{array}$ & $\begin{array}{l}\text { There is no specific } \\
\text { control mechanism. }\end{array}$ & $\begin{array}{l}\text { There is a control } \\
\text { unit, which monitors } \\
\text { all the activities of } \\
\text { processing. }\end{array}$ \\
\hline Learning & $\begin{array}{l}\text { They can endure } \\
\text { uncertainty }\end{array}$ & $\begin{array}{l}\text { Very particular, } \\
\text { structured } \\
\text { formatted data is } \\
\text { needed to endure } \\
\text { uncertainty }\end{array}$ \\
\hline $\begin{array}{l}\text { Fault } \\
\text { tolerance }\end{array}$ & $\begin{array}{l}\text { Execution degrades } \\
\text { with even partial } \\
\text { damage }\end{array}$ & $\begin{array}{l}\text { It is able of robust } \\
\text { performance, hence } \\
\text { has the expected to be } \\
\text { fault tolerant }\end{array}$ \\
\hline
\end{tabular}

\section{METHODOLOGY ADOPTED FOR} HEALTHCARE SYSTEM

There are many types of Artificial Neural Networks. Major ones are as follows -

\subsection{Perceptron Neural Network}

A perceptron neural network (PNN) does certain calculations to notice features of business intelligence in the input data. This network is used in fault detection system [1]. PNN was first introduced by Frank Rosenblatt in 1957. He proposed a Perceptron learning rule based on the original MCP (McCulloch-Pitts) neuron.

A Perceptron is an algorithm for supervised acquisition of binary classifiers. This algorithm empowers neurons to learn and measures components in the preparation set once at a time. 


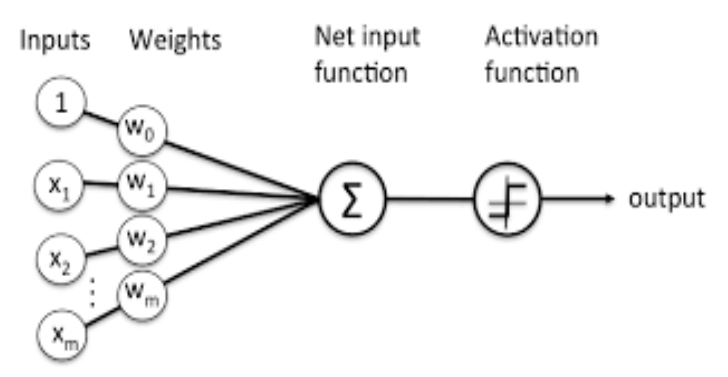

Fig. 4 Perceptron neural network

There are two types of Perceptron- Single layer and Multilayer Perceptron.

[1] Single layer Perceptron can learn just straight detachable patterns.

[2] Multilayer Perceptron or feedforward neural network has two or more layers with the greater processing power.

The Perceptron algorithm find out the weights for the input signals in order to draw a linear decision limit. This empowers us to differentiate between the two linear divisible classes +1 and -1 .

\subsection{Feedforward Neural Network}

The feedforward neural network was the first and simple type of network, where the input data goes one way. The data goes through the input nodes and exit on the output nodes. Feedforward neural network may have the hidden layers.

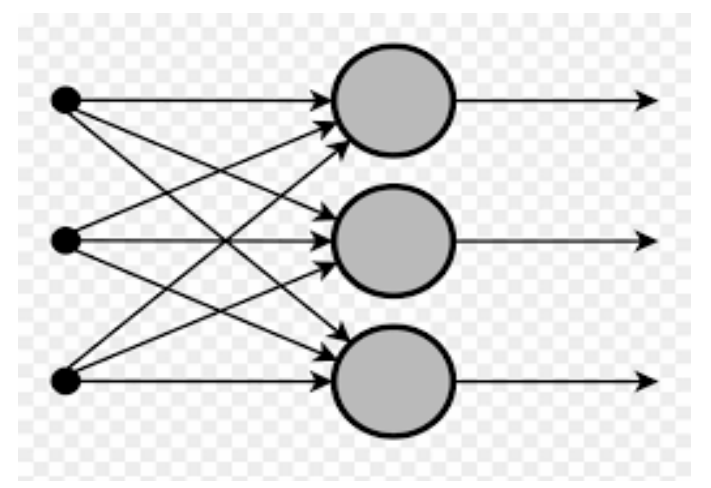

Fig. 5 Feedforward neural network

Feedforward neural network is appropriate in signature confirmation, image processing and speech recognition where characterizing the target classes are complex [2] .

\subsection{Recurrent Neural Network}

The Recurrent Neural Network (RNN) works on the rule of saving the output of a layer and returning to the input to help in anticipating the final result of the layer.

Presently, the first layer is characterized like the feedforward neural network with result of the sum of the weights and highlights. At the point when RNN process begins calculation, it implies that starting with one stage then onto the next every neuron will recover some data it had in the past. Here each neuron acts as a memory cell for performing ccalculation. In this process, it generally let the neural network to work on the front propagation and recover some data it required later. Presently, if the prediction isn't right it uses the learning rate or error correction to create little alteration so that it will delinquently pursue towards making the accurate forecasting during the back-propagation.

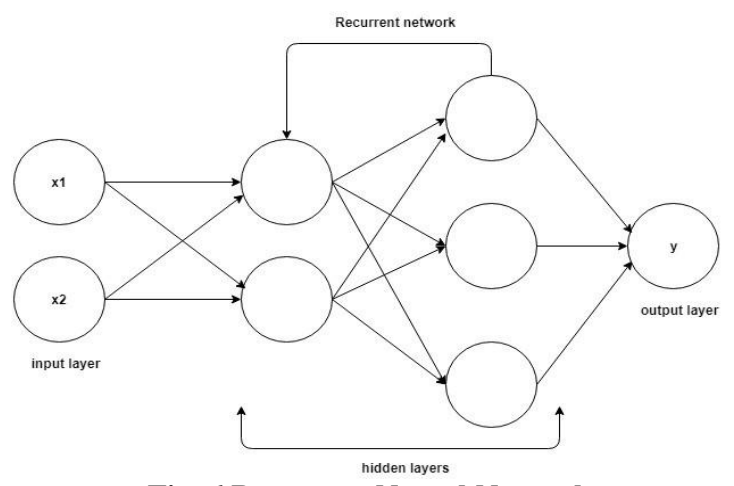

Fig. 6 Recurrent Neural Network

Recurrent Neural Networks are applicable in rhythm learning and speech reorganization [3].

\subsection{Modular Neural Network}

Modular Neural Networks (MNNs) is a fast developing territory in artificial neural network research. MNNs have a lot of different networks working independently and contributing towards the output. Each neural network has a variety of inputs which are unambiguously differentiated to various networks building and performing sub-tasks. These networks don't interface with each other in achieving the tasks. The advantages of a MNN is that it breakdowns a large process into many modules for declining the unpredictability.

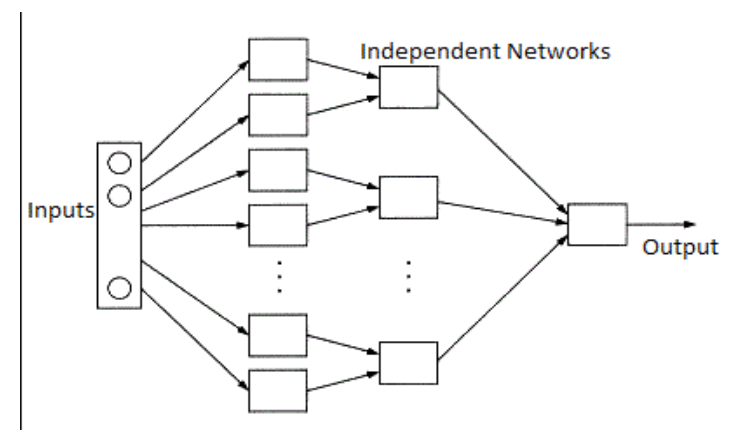

Fig. 7 Modular neural network

Modular Neural Networks are applicable in numerous regions, for example face recognition and stock market prediction [4].

\subsection{Back-propagation Neural Network}

A neural network has a variety of connected input-output units where every association has a weight related with its computer programs. It helps to frame predictive models from large database.

Back-propagation is the technique of calibrating the weights of a neural network depend on the error rate obtained from the previous iteration. Proper tuning of the weights allow you to decrease error rate and create the reliable model. This technique helps us to calculate the function with all the weights within the network.

Back-propagation is particularly helpful for deep neural networks running on error-prone activity like speech or image recognition, ocean tidal prediction [5] etc.

Consider the following diagram - 


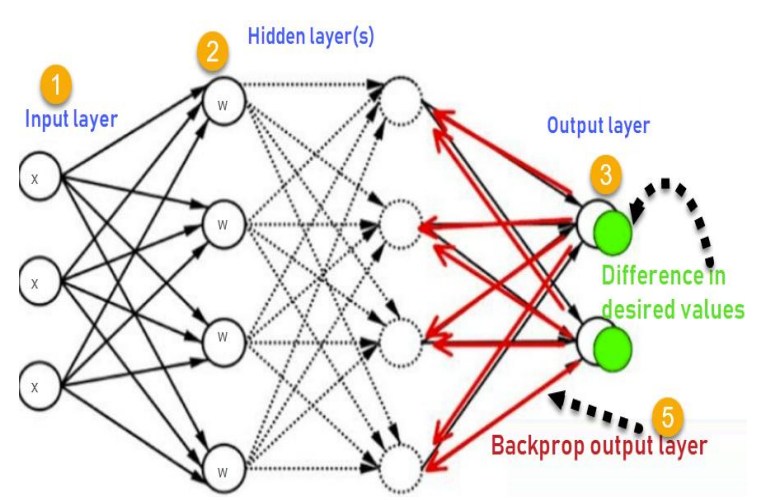

Fig. 8 Back-propagation neural network

1. Input $\mathrm{X}$, overcome the preconnected way.

2. Input $\mathrm{X}$ is shown using real weights $\mathrm{W}$. The weights are typically randomly chosen.

3. Compute the output for each neuron from the input layer to the hidden layers and output layer.

4. Compute the mistake in the outputs as

Mistake $=$ Actual Output-Desired Output

5. Travel back from the output layer to the hidden layer to coordinate the weights such that the mistake is diminished.

Keep repetition the method until the desired output is obtained.

There are two types of Back-propagation Networks -

- $\quad$ Static Back-propagation

- Recurrent Back-propagation

Static back-propagation: This type of back-propagation network creates a planning of a static input for static output. It is significant in static characterization, for instance, optical character recognition.

Recurrent Back-propagation: This type of back-propagation network feed forward until a fixed value is obtained. At this point, the mistake is determined and propagated backward.

The essential difference between both of above networks is that the planning is quick in static back-propagation while it is non-static in recurrent back-propagation [6].

\section{CONCLUSION}

This study expected to evaluate artificial neural network in healthcare system. ANNs play a vital role in the healthcare field in solving various health problems. Applications of ANN are mainly found to be classification, prediction, and diagnosis. The goal of this paper is to introduce an exposure of the role of ANNs in healthcare system. ANN is used as a powerful tool to help physicians for satisfactory diagnosis of various diseases. Roles of the some artificial neural networks in healthcare system are as follows-

i. A perceptron neural network used in fault detection system.

ii. Feed forward neural networks are applicable in image processing, Signature verification and speech recognition.

iii. The Recurrent neural networks can also used in speech reorganization.

iv. Modular neural networks are applicable in stock market prediction and face recognition.

v. Back-propagation is particularly useful for image or speech recognition and long term ocean tidal prediction.

vi. Many academics are trying to solve complicated problems that were unexplained before using ANN. For examples clinical imaging which help doctors in interpretation MRIs and Genomics where DNA chains are read.

\section{REFERENCES}

[1] Marcin Mrugalski, M. W., 2008. Confidence estimation of the multi-layer perceptron and its application in fault detection systems. ELSEVIER , pp. 895-906.

[2] J.P. Drouhard, R. S., 1996. A Neural Network Approach to Off-Line Signature Verification using Directional PDF. ESLEVIER , pp. 415-424.

[3] Felix Weninger, H. E., 2015. Speech Enhancement with LSTM Recurrent Neural Networks and its Application to Noise-Robust ASR. Springer International Publishing ,pp. 91-95.

[4] Patricia Melin, O. M., 2011. Face Recognition With an Improved Interval Type-2 Fuzzy Logic Sugeno Integral and Modular Neural Networks. IEEE , pp. 1001-1012.

[5] Lee, T.-L., 2004. Back-propagation neural network for long-term tidal predictions. ELSEVIER, pp. 225-238.

[6] 6.https://www.guru99.com/backpropogation-neuralnetwork.html

[7] Filippo Amato, A. L.-M., 2013. Artificial neural networks in medical diagnosis. Journal of Applied Biomedicine, pp. 47-58.

[8] Verma, M., 2014. Medical Diagnosis using Back Propagation Algorithm in ANN . International Journal of Science, Engineering and Technology Research (IJSETR), Volume 3, Issue 1, pp. 94-99.

[9] Pattichis, C. S., 2001. Adaptive Neural Network Imaging in Medical Systems. IEEE , pp. 313-317. 